\title{
Terapia por contensão induzida: revisão de ensaios clínicos
}

\section{Constraint-induced movement therapy: reviews of clinical trials}

\author{
Lidiane de Araújo Silva ${ }^{[a]}$, Vanessa Tamashiro ${ }^{[b]}$, Rodrigo Deamo Assis ${ }^{[c]}$
}

[a] Fisioterapeuta graduada pela Universidade São Marcos (UNIMARCO), São Paulo, SP - Brasil, e-mail: lilidycat@yahoo.com.br

[b] Fisioterapeuta graduada pela Universidade São Marcos (UNIMARCO), São Paulo, SP - Brasil, e-mail: vanessatamashiro@yahoo.com.br

[c] Fisioterapeuta, Doutor pelo Departamento de Neurologia/Neurocirurgia da Universidade Federal de São Paulo (UNIFESP/ EPM), São Paulo, SP - Brasil, e-mail: rodrigodeamo@neuro.epm.br

\section{Resumo}

Introdução: A Terapia por Contensão Induzida (TCI) é uma terapêutica que visa à recuperação da função do membro superior (MS) parético de pacientes com sequelas de um acidente vascular cerebral por meio de treinamento intensivo, uso de uma restrição no MS não-parético durante 90\% do dia e pelo pacote de transferência. Objetivo: O objetivo deste trabalho é realizar uma revisão entre os estudos que compararem a TCI com outras terapêuticas. Metodologia: Foi realizada uma pesquisa nas bases de dados MEDLINE, SciELOo e ISI Web of Science com o descritor "constraint induced movement therapy" durante o mês de junho de 2008. Resultados: Foram encontrados seis artigos onde a TCI foi comparada com os métodos Bobath e Kabat. Discussão: Dentre os artigos encontrados, percebemos que há uma variação entre as amostras de cada estudo, assim como o método de avaliação entre as terapêuticas. Conclusão: A TCI mostrou-se superior em relação às outras terapêuticas.

Palavras-chave: Terapia por contensão induzida. Bobath. Kabat. Reabilitação.

\section{Abstract}

Introduction: The Constraint-induced Movement Therapy (CIMT) is a therapeutic which main goal is the functional recuperation of paretic upper extremity (UE) of stroke survivors by an intensive treatment, wear of restriction in non-paretic UE during $90 \%$ of activity day and by for the transfer package. Objective: The main of this paper is to do a review between studies which compare the CIMT between other therapeutics. Methods: Had been made an search between MEDLINE, SciELO and ISI Web of Science with the principal word "constraint induced movement therapy" during the month of June of the 2008. Results: We founded six articles where the CIMT was compared with the therapeutics Bobath and 
Kabat. Discussion: Inside the articles founded, we observed a variation between the population in each study, as by the outcomes to evaluate the therapeutics. Conclusion: The CIMT showed itself superior by the other therapeutics.

Keywords: Constraint-induced movement therapy. Bobath. Kabat. Rehabilitation.

\section{Introdução}

Segundo a Organização Mundial de Saúde, o Acidente Vascular Cerebral (AVC) é a terceira maior causa de morte no mundo depois das doenças cardíacas e do câncer. Além de ser a principal causa de incapacidade nos Estados Unidos, com aproximadamente 730.000 casos por ano (1, 2), e na América Latina, o AVC encontra-se também entre os principais problemas de saúde pública, apesar de os poucos estudos epidemiológicos existentes não quantificarem a real dimensão do problema $(3,4)$.

A Terapia por Contensão Induzida (TCI) "Constraint-induced Movement Therapy", também conhecida como Técnica de Restrição, é uma nova terapêutica que visa recuperar a função do membro superior (MS) parético de pacientes com sequelas motoras de lesões encefálicas adquiridas por meio de treinamento intensivo e uso de uma restrição, luva ou tipoia, no MS não-parético durante $90 \%$ do dia (5).

A TCI teve o seu início com pesquisas pré-clínicas em primatas machos jovens $(6,7)$ e possui embasamento teórico pela superação da Teoria do Desuso "learned nonused", pela qual os primatas voltaram a utilizar o MS parético nas atividades cotidianas após o uso forçado do MS não-parético durante duas semanas (8-10).

Atualmente o protocolo mais utilizado da TCI é o de duas semanas consecutivas, com 6 horas diárias de prática supervisionada "shaping procedures e task pratice", com o uso de uma restrição durante $90 \%$ do dia e realização do pacote de transferência, que consiste no contrato comportamental, aplicação diária da avaliação "Motor Activity Log” e prática de exercícios domiciliares (11, 12). Esta terapêutica tem-se mostrado eficaz em estudos envolvendo populações com AVC (13-20), traumatismo crânio-encefálico (21), paralisia cerebral (22) e em estudos que envolvam técnicas de imagem para comprovar a reorganização cortical (23-26).

Este estudo tem como objetivo realizar uma revisão de artigos que comparem a TCI com outras técnicas de reabilitação.

\section{Metodologia}

Foi realizada uma revisão bibliográfica durante o mês de junho de 2008 nas bases de dados: MEDLINE, SciELO e ISI Web of Science com o descritor "constraint induced movement therapy". Os critérios de inclusão foram: (a) ano de publicação entre 2000 e 2008 e (b) estudos que compararam a TCI com outras terapêuticas por meio de ensaios clínicos controlados e randomizados. O critério de exclusão foi a associação da TCI com outras técnicas de reabilitação.

\section{Resultados}

Foram encontrados 352 artigos, dos quais apenas 6 (27-32) supriram os critérios de inclusão e exclusão. Dos artigos selecionados, as técnicas comparadas com a TCI foram: Conceito Neuroevolutivo (método Bobath) e Facilitação Neuromuscular Proprioceptiva (método Kabat).

Os artigos foram analisados em relação ao ano de publicação, metodologia, testes utilizados para mensurar o ganho motor do MS parético, conclusão dos autores e país de origem, cujos resultados estão ilustrados no Quadro 1: 
Quadro 1 - Análise descritiva dos resultados

\begin{tabular}{|c|c|c|c|c|c|}
\hline AUTOR & ANO & METODOLOGIA & TESTES & CONCLUSÃO & $\begin{array}{l}\text { PAÍS DE } \\
\text { ORIGEM }\end{array}$ \\
\hline Areeat S, et al. (27) & 2004 & $\begin{array}{c}\text { TCI } n=33 \\
\text { Bobath } n=36\end{array}$ & ARAT & $\begin{array}{l}\text { TCI apresentou-se } \\
\text { mais eficaz com } \\
\text { relação ao Bobath } \\
\text { de acordo com a } \\
\text { escala utilizada para } \\
\text { avaliação }\end{array}$ & TAILÂNDIA \\
\hline Page SJ,.et al. (28) & 2004 & $\begin{array}{c}\text { TCI } n=7 \\
\text { FNP } n=4 \\
\text { nenhuma terapia } n=6\end{array}$ & $\begin{array}{c}\text { FMA, ARAT, } \\
\text { MAL }\end{array}$ & $\begin{array}{l}\text { TCI apresentou-se } \\
\text { mais eficaz com } \\
\text { relação ao FNP em } \\
\text { todas as escalas } \\
\text { utilizadas para } \\
\text { avaliação }\end{array}$ & EUA \\
\hline Wolf S, et al. (29) & 2006 & $\begin{array}{l}\text { TCI } n=106 \\
\text { FC } n=116\end{array}$ & WMFT,MAL & $\begin{array}{l}\text { TCI apresentou-se } \\
\text { mais eficaz com } \\
\text { relação à FC em } \\
\text { todas as escalas } \\
\text { utilizad as para } \\
\text { avaliação }\end{array}$ & EUA \\
\hline Wu C, et al. (30) & 2007 & $\begin{array}{c}\text { TCI } n=23 \\
\text { Bobath } n=24\end{array}$ & $\begin{array}{l}\text { FMA, MAL, } \\
\text { Kinematic Of } \\
\text { Reaching } \\
\text { Movement Used } \\
\text { To Describe } \\
\text { The Control } \\
\text { Strategies For } \\
\text { Reaching }\end{array}$ & $\begin{array}{l}\text { TCI apresentou-se } \\
\text { mais eficaz com } \\
\text { relação ao Bobath } \\
\text { em todas as escalas } \\
\text { utilizadas para } \\
\text { avaliação }\end{array}$ & CHINA \\
\hline Wu C, et al. (31) & 2007 & $\begin{array}{c}\text { TCI } n=13 \\
\text { Bobath } n=13\end{array}$ & $\begin{array}{l}\text { FMA, MAL, } \\
\text { Stroke Impact } \\
\text { Scale }\end{array}$ & $\begin{array}{l}\text { TCI apresentou-se } \\
\text { mais eficaz com } \\
\text { relação ao Bobath } \\
\text { em todas as escalas } \\
\text { utilizadas para } \\
\text { avaliação }\end{array}$ & CHINA \\
\hline Gauthier LV, et al (32) & 2008 & $\begin{array}{l}\text { TCI } n=16 \\
\text { FC } n=20\end{array}$ & $\begin{array}{c}\text { Ressonância } \\
\text { Magnética } \\
\text { Funcional }\end{array}$ & $\begin{array}{l}\text { TCI mostrou-se } \\
\text { capaz de incentivar } \\
\text { a produção de } \\
\text { novas conexões } \\
\text { sinápticas }\end{array}$ & EUA \\
\hline
\end{tabular}

Legenda: TCI - Terapia por Contensão Induzida; n - amostra do estudo; ARAT - Action Research Arm Test; FMA - Fugl Mever Avaliation; WMFT - Wolf Motor Funtion Test; FC - fisioterapia convencional (estudos que não descriminam qual tipo de intervenção foi realizada); e MAL - Motor Activity Log 


\section{Discussão}

É importante destacarmos os principais problemas metodológicos deste estudo:

a) há poucos artigos que comparam a TCI com outra técnica de reabilitação;

b) dentre os artigos encontrados, com exceção do estudo EXCITE, o número da amostra é pequeno; e

c) os artigos somente foram pesquisados em três bases de dados.

É interessante observar o número tão pequeno de artigos que comparem a TCI com outras terapêuticas encontrado dentre a grande quantidade de artigos. É possível que haja grande quantidade de estudos sobre o tema, pois a TCI possui um protocolo de atividades de fácil replicação e é uma terapêutica nova, e o número reduzido de estudos comparativos se deve à falta de tempo para a aplicação do protocolo e também desconhecimento da terapêutica por parte dos profissionais (33).

Os resultados demonstram que: (a) o país que mais pesquisa a TCI é o EUA, (b) os testes mais utilizados são o "Wolf Motor Function Test" e o "Action Research Arm Test" e (c) as técnicas mais utilizadas para comparação com a TCI foram o tratamento neuroevolutivo e a facilitação neuromuscular proprocpetiva.

Acreditamos que os EUA sejam o principal país a pesquisar a TCI, pois a terapia é de origem norte-americana, e que a comparação entre as técnicas Bobath e Kabat foi encontrada por serem técnicas mais antigas.

Atualmente, as técnicas mais utilizadas na reabilitação neurológica são o "Bobath" e o "Kabat", as quais ganharam grande credibilidade por serem pioneiras na área. Um fator dificultador dessas terapias é não haver um protocolo a ser seguido, pois a TCI possui tarefas estruturadas e seus resultados podem ser mascarados pela associação a outros tipos de terapias, entre elas, cinesioterapia, eletroestimulação ou hidroterapia. Além disso, são técnicas que possuem pouca comprovação científica e os resultados em relação à melhora funcional do paciente podem demorar anos para serem obtidos.

O Tratamento Neuroevolutivo "Bobath" é uma das abordagens mais utilizadas em países como China e Brasil, embora esse método de tratamento nunca tenha sido comprovado como sendo mais eficaz do que outras modalidades. No entanto, no estudo (27) em que a TCI foi comparada com o Bobath, observou-se que os resultados das duas técnicas são eficazes com relação à melhora da destreza do membro superior, porém somente a TCI apresentou melhoras significativas na força de preensão e de pinça.

Além do Bobath, outra técnica também muito utilizada é a Facilitação Neuromuscular Proprioceptiva "Kabat", baseada em diagonais funcionais do movimento que, apesar de demonstrar alguns benefícios, apresentou-se pouco eficaz quando comparada à TCI, pela pouca funcionalidade em relação às atividades de vida diárias e, assim como o Bobath, por ser um tratamento para longo prazo.

Um fator que nos chamou a atenção foi a quantidade de artigos relacionados com o tema TCI, pois a terapia passou a ser estudada na década de $80 \mathrm{e}$, em comparação com as terapêuticas anteriormente citadas, é uma técnica recente, indicando que o treinamento intensivo é o caminho correto da reabilitação $(34,35)$.

É importante percebemos que a TCI é amplamente estudada e mostrou-se superior às outras terapêuticas, porém é difícil comparar os resultados dos artigos selecionados entre si, por haver variação em relação ao número de horas de prática supervisionada e em cada estudo utilizou-se um tipo específico de avaliação.

No Brasil, a TCI é considerada uma nova terapêutica, utilizada somente em alguns centros de reabilitação, tais como a Universidade Federal de São Paulo (UNIFESP) e Universidade São Paulo (USP), e este diminuto interesse em sua aplicação prática pode ser em decorrência de: (a) a TCI ser realizada de forma individual, (b) haver grande demanda de pacientes com sequelas de AVC para esses centros de reabilitação e $(C)$ o fator custo-benefício ser ainda pouco estudado.

Em nosso país há poucos profissionais especializados nesse tratamento e poucos artigos (36-40) concentrados na TCI, porém percebe-se crescente interesse da comunidade científica sobre esse tema, por isso sugere-se que novos estudos envolvendo a TCI sejam realizados, tais como: estudos com casos controlados ou com a redução da carga horária da prática supervisionada. 


\section{Conclusões}

Todas as terapêuticas apresentam melhora da função do MS parético em pacientes com sequelas motoras de um AVC, contudo, a TCI é uma técnica que tem contribuído para esclarecer as possíveis teorias da recuperação neurológica. Para a implicação na pesquisa, este estudo demonstrou haver grande variedade de artigos com o tema, porém escassez em estudos clínicos. Quanto à prática, foi demonstrado ser possível utilizar a TCI como tratamento adjunto à reabilitação convencional.

\section{Referências}

1. Feigin VL, Lawes CM, Bennett DA, Anderson CS. Stroke epidemiology: a review of population-based studies of incidence, prevalence, and case-fatality in the late 20th century. Lancet Neurol. 2003;2(1):43-53.

2. Strong K, Mathers C, Bonita R. Preventing stroke: saving lives around the world. Lancet Neurol. 2007;6(2):182-7.

3. Lotufo PA. Stroke in Brazil: a neglected disease. Sao Paulo Med J. 2005;123(1):3-4.

4. Saponisk G, Del Brutto OH. Stroke in South America: a systematic review of incidence, prevalence and stroke subtypes. Stroke. 2003;34(9):2103-8.

5. Wolf S, Blanton S, Baer H, Breshears J, Butler AJ. Repetitive task practice: a critical review of constraintinduced movement therapy in stroke. Neurologist. 2002;8(6):325-38.

6. Taub E. Movement in nonhuman primates deprived of somatosensory feedback. In: Keogh JF, editor. Exercise and sports science reviews. Santa Barbara: Journal Publishing Affiliates; 1977. p. 335-74.

7. Tower SS. Pyramidal lesions in monkeys. Brain. 1940;63:36-90.

8. Taub E, Uswatte G. Constraint-induced movement therapy: brindging from the primate laboratory to the stroke rehabilitation laboratory. J Rehabil Med. 2003;(41 Suppl):34-40.

9. van der Lee, JH. Constraint-induced movement therapy: some thoughts about theories and evidence. J Rehabil Med. 2003;(41 Suppl):41-5.

10. Taub E, Uswatte G, Mark VW, Morris DM. The learned nonuse phenomenon: implications for rehabilitation. Eura Medicophys. 2006;42(3):241-56.

11. Morris DM, Taub E, Mark VW. Constraint-induced movement therapy: characterizing the intervention protocol. Eura Medicophys. 2006;42(3):257-68.

12. Taub E, Miller NE, Novack TA, Cook EW, Fleming WC, Nepomuceno CS, et al. Technique to improve chronic motor defict after stroke. Arch Phys Med Rehabil. 1993;74(4):347-54.

13. Wolf SL, Lecraw DE, Barton LA, Jann BB. Forced use of hemiplegic upper extremities to reverse the effect of learned nonuse among chronic stroke and head-injured patients. Exp Neurol. 1989;104(2):125-32.

14. Sabari JS, Kane L, Flanagan SR, Steinberg A. Constraint-induced motor relearning after stroke: a naturalistic case report. Arch Phys Med Rehabil. 2001;82(4):524-8.

15. Barreca S, Wolf SL, Fasoli S, Bohannon R. Treatment interventions for the paretic upper limb of stroke survivors: a critical review. Neurorehabil Neural Repair. 2003;17(4):220-6.

16. Taub E, Uswatte G, King DK, Morris D, Crago JE, Chatterjee A. A placebo-controlled trial of constraintinduced movement therapy for upper extremity after stroke. Stroke. 2006;37:1045-49. 
17. Sterr A, Szameitat A, Shen S, Freivogel S. Application of the CIT concept in the clinical environment: hurdles, practicalities, and clinical benefits. Cogn Behav Neurol. 2006;19(1):48-54.

18. Page SJ, Levine P. Modified constraint-induced movement therapy extension: using remote technologies to improve function. Arch Phys Med Rehabil. 2007;88(7):922-7.

19. Dettmers C, Teske U, hamzei F, Uswatte G, Taub E, Weiller C. Distributed form of constraint-induced movement therapy improves functional outcome and quality of life after stroke. Phys Med Rehabil. 2005;86:204-9.

20. Kunkel A, Kopp B, Muller G, Villringer K, Villringer A, Taub E, et al. Constraint-induced movement therapy for motor recovery in chronic stroke patients. Phys Med Rehabil. 1999;80:624-28.

21. Morris D, Shaw SE, Mark VW, Uswatte G, Barman J, Taub E. The influence of neuropsychological characteristics on the use of CI therapy with persons traumatic brain injury. NeuroRehabilitation. 2006;21(2):131-7.

22. DeLucca SC, Echols K, Ramey SL, Taub E. Pediatric constraint-induced movement therapy for a young child with cerebral palsy: two episodes of care. Phys Ther. 2003;83(11):1003-13.

23. Liepert J, Miltner WHR, Bauder H, Sommer M, Dettmers C, Taub E, et al. Motor cortex plasticity during constraint-induced movement therapy in stroke patients. Neurosci Lett. 1998;250:5-8.

24. Hamzei F, Liepert J, Dettmers C, Weiller C, Rijntjes M. Two different reorganizastion patter after rehabilitative therapy: an exploratory study wiht fMRI and TMS. Neuroimage. 2006;31:710-20.

25. Cramer SC, Nelles G, Benson RR. A functional MRI study of subjects recovered from hemiparetic stroke. Stroke. 1997;28(12):2518-27.

26. Park S, Butler AJ, Cavalheiro V, Alberts JA, Wolf SL. Changes in serial optical topography and TMS during task performance after constraint-induced movement therapy in stroke: a case study. Neurorehabil Neural Repair. 2004;18:95-105.

27. Areeat S, Nijasri CS. Effectiveness of constraint-induced movement therapy in chronic stroke patients. J Med Assoc Thais. 2004;87:148-55.

28. Page SJ, Sisto S, Levine P, McGrath RE. Efficacy of modified constraint-induced movement therapy in chronic stroke: a single blinded randomized controlled trial. Arch Phys Med Rehabil. 2004;85(1):14-8.

29. Wolf SL, Winstien CJ, Miller JP, Taub E, Uswatte G, Morris D, et al. Effect of constraint-induced movement therapy on upper extremity function 3 to 9 months after the stroke the excite randomized trial. JAMA. 2006;296(17):2095-104.

30. Wu C, Chen C, Tsai W, Lin K, Chou S. A randomised controlled trial of modified constraint induced movement therapy for elderly stroke survivors: changes in motor impairment daily functioning, and quality of life. Arch Phys Med Rehabil. 2007;88(3):273-8.

31. Wu C, Chen C. Kinematic and clinical analyses of upper-extremity movements after constraint-induced movement therapy in patients with stroke: a randomized controlled trial. Arch Phys Med Rehabil. 2007;88(8):964-70.

32. Gauthier LV, Taub E, Perkins C, Ortmann M, Mark VW, Uswatte G. Remodeling the brain: plastic structural brain changes produced by different motor therapies after stroke. Stroke. 2008;39(5):1520-5.

33. Page SJ, Levine P, Sisto S, Bond Q, Johnston MV. Stroke patients and therapists opinions of constraintinduced movement therapy. Clin Rehabil. 2002;16(1):55-60. 
34. Trakka IM, Kononen M, Pitkanen K, Sivenius J, Mervaalat E. Alterations in cortical excitability in chronic stroke after constraint-induced movement therapy. Neurol Res. 2008;30(5):504-10.

35. Richards L, Senesac C, McGuirk T, Woodbury M, Howland D, Davis S et al. Response to intensive upper extremity therapy by individuals with ataxia from stroke. Top Stroke Rehabil. 2008;15(3):262-71.

36. Riberto M, Monroy HM, Kaihami HN, Otsubo PPS, Battistella LR. A terapia de restrição como forma de aprimoramento do membro superior em pacientes com hemiplegia. Acta Fisiatr. 2005;12(1):15-9.

37. Souza WC, Conforto AB, Charles AD. Terapia de restrição e indução do movimento em pacientes pós AVC. Fisioter Bras. 2007;8:65-8.

38. Assis RD, Massaro AR, Chamlian TR, Silva MF, Ota S. Terapia de restrição para uma criança com paralisia cerebral com hemiparesia: estudo de caso. Acta Fisiatr. 2007;14(1):62-5.

39. Assis RD, Chamilian TR, Ferreira MS, Massaro AR. Terapia por contensão induzida: um estudo exploratório. Med Reabil. 2008;27:45-8.

40. Diniz L, Abranches M. Neuroplasticidade na terapia de restrição e indução do movimento em pacientes com acidente vascular encefálico. Med Reabil. 20003;22:53-5.

Recebido: 26/01/2009

Received: 01/26/2009

Aprovado: $10 / 11 / 2009$

Approved: 11/10/2009 